Case Report

\title{
Prenatal Identification and Molecular Characterization of Two Simultaneous De Novo Interstitial Duplications of Chromosomal Regions 7p22.1p21.1 and 15q24.1
}

\author{
Sabrina C. Burn $\mathbb{D}^{1,2,3}$ Kali Swift, ${ }^{3}$ and Maria Palmquist ${ }^{2,3}$ \\ ${ }^{1}$ Department of Obstetrics, Gynecology \& Women's Health, University of Minnesota, Minneapolis, MN, USA \\ ${ }^{2}$ Sanford School of Medicine, The University of South Dakota, Sioux Falls, SD, USA \\ ${ }^{3}$ Division of Maternal Fetal Medicine, Avera McKennan Hospital \& University Health Center, Sioux Falls, SD, USA
}

Correspondence should be addressed to Sabrina C. Burn; burn@umn.edu

Received 25 September 2017; Accepted 14 January 2018; Published 11 February 2018

Academic Editor: Philip D. Cotter

Copyright @ 2018 Sabrina C. Burn et al. This is an open access article distributed under the Creative Commons Attribution License, which permits unrestricted use, distribution, and reproduction in any medium, provided the original work is properly cited.

\begin{abstract}
The occurrence of simultaneous de novo chromosomal aberrations is extremely rare. Here, we describe two, previously unreported, simultaneous de novo interstitial duplications of chromosomes $7 \mathrm{p}$ and 15q. Amniocentesis was completed for a healthy gravida 4 para 3 woman due to her advanced maternal age and concurrent ultrasound findings of partial vermian agenesis, choroid-plexus cysts, and hypoplastic nasal bone. Cytogenetic analysis of cultured amniocytes by conventional chromosome analysis, comparative genomic hybridization, and fluorescence in situ hybridization revealed two interstitial duplications of the chromosomal regions 7p22.1p21.1 and 15q24.1, leading to partial trisomy of 7p and 15q and karyotype 46,XY,dup(7)(p22.1-p21.1),dup (15)(q24.1). Parental chromosomal analysis did not identify any heritable changes, suggesting both mutations were de novo in nature. Postnatal examination of the neonate was significant for low set ears, thick helices, flat nasal bridge, ankyloglossia, and aberrant head shape and size concerning for craniosynostosis. Postnatal MRI was consistent with Dandy-Walker variant showing hypogenesis of the inferior cerebellar vermis. To our knowledge, there are no prenatal or postnatal reports of comparable duplications involving these two regions simultaneously. Continued observation of the neonate may reveal further phenotypic consequences of these two simultaneous de novo interstitial duplications.
\end{abstract}

\section{Introduction}

It is well documented that the probability of chromosomal aberrations increases significantly with maternal age. An abnormal ultrasound accompanying advanced maternal age is significant and is most often followed by further genetic testing. The implementation and complementary use of conventional and newer molecular cytogenetic techniques have enabled diagnostic and prognostic parental counseling. As these techniques have steadily improved it is now possible to locate and characterize the aberration and identify potential consequences of such mutations. However, it is still difficult to provide adequate counseling in the absence of any prenatal or postnatal reports of similar chromosomal aberrations or any previously identified phenotypes. This is true in particular for previously unidentified and uncharacterized de novo interstitial duplications whose occurrence is relatively rare. Here, we report the prenatal identification and molecular characterization of two, previously unreported, simultaneous de novo interstitial duplications of chromosomal regions $7 \mathrm{p} 22.1 \mathrm{p} 21.1$ and $15 \mathrm{q} 24.1$ with associated phenotype using a combination of advanced ultrasonography, conventional chromosome analysis, microarray comparative genomic hybridization (CGH), and fluorescence in situ hybridization (FISH) analysis.

\section{Clinical Report and Cytogenetic Analysis}

2.1. Initial Examination. An otherwise healthy 37-year-old, gravida 4 para 3, woman was referred to our center after the identification of choroid-plexus cysts and concern for additional brain anomaly arose on her 20-week ultrasound scan. 


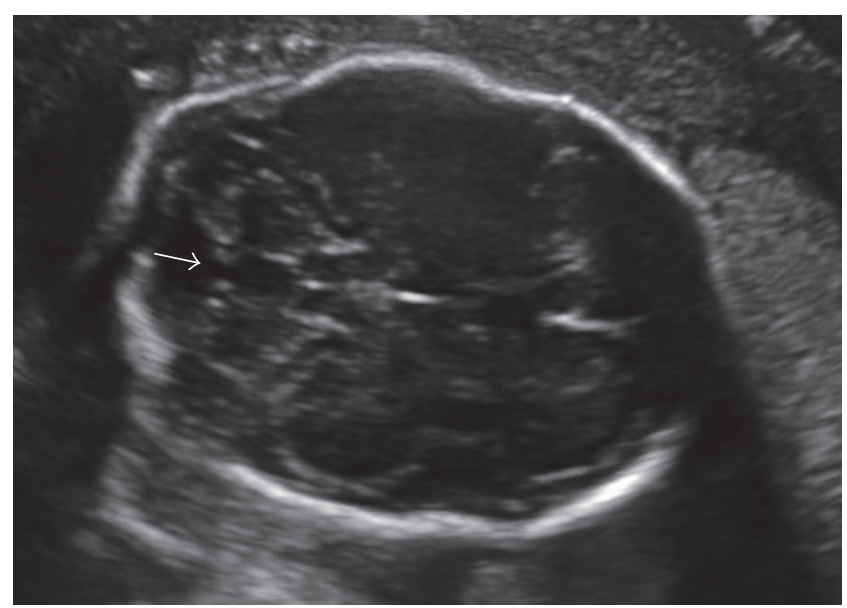

(a)

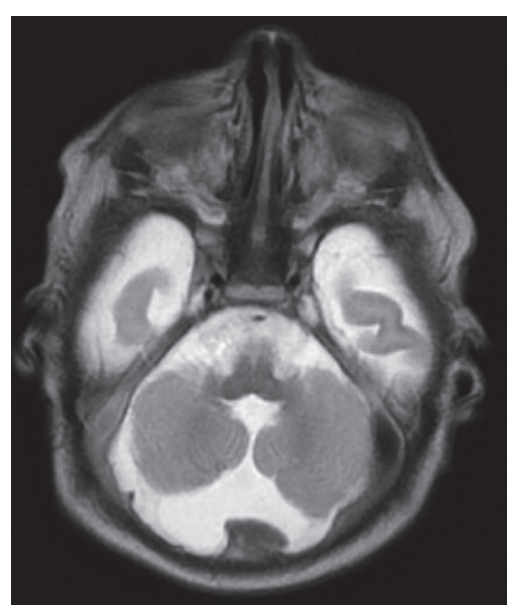

(b)

FIGURE 1: (a) Prenatal axial ultrasound shows the skull shape aberrancy and the presence of vermian agenesis with communication between the 4th ventricle and the cisterna magna (depicted by arrow). (b) Postnatal MRI was significant for hypogenesis of the inferior cerebellar vermis with mild fourth ventricular enlargement which communicates with a retrocerebellar CSF collection.

Complete sonographic examination at 21-week gestation identified multiple abnormalities, including partial vermian agenesis (formerly referred to as Dandy-Walker variant), hypoplastic nasal bone, choroid-plexus cysts, and dangling choroid (Figure 1(a)). The identification of multiple fetal anomalies suggested an increased likelihood of aneuploidy or other genetic abnormalities. The patient's advanced maternal age and brother with Down syndrome further indicated the possibility of a cytogenetic abnormality. Due to these factors, the patient elected to proceed with genetic amniocentesis.

2.2. Amniocentesis and Conventional Chromosome Analysis. Amniocentesis results were as follows: (i) Precision Panel analysis was performed via the Luminex xMap Technology of 20 known genetic syndromes and regions to identify the presence of aneuploidies in chromosomes 13, 18, 21, X, and Y. The Panel identified no abnormalities in the DNA loci tested. (ii) Conventional chromosomal analysis on amniotic fluid revealed an abnormal 46,XY,add(7)(p15) karyotype. More specifically, an abnormal chromosome complement characterized by the presence of additional quantifiable material of unknown origin was identified at band $7 \mathrm{p} 15$ in each metaphase cell analyzed.

2.3. Microarray Comparative Genomic Hybridization (CGH) Analysis. To further characterize the breakpoint region, estimate size, and approximate number of genes located within the $7 \mathrm{p} 15$ region, a microarray CGH analysis was performed. Analysis of chromosome 7 confirmed the presence of an interstitial gain of the $7 \mathrm{p} 22.1 \mathrm{p} 21.1$ region with genomic coordinate chr7:5262454-20572298. The gain was approximately $15.31 \mathrm{Mb}$ in size. Genoglyphix ${ }^{\circledR}$ Analysis software suggests this area of duplication is comprised of at least 72 genes, of which 43 are OMIM genes. The result is a clinically relevant alteration, which results in partial trisomy of 7p22.1p21.1. Unexpected was the detection of another clinically significant interstitial gain in chromosome 15 . The interstitial gain was found in the 15q24.1 region with genomic coordinate chr15:73775826-75162902 and was approximately $1.39 \mathrm{Mb}$ in size. This region contains approximately 31 genes, including 22 OMIM genes. Based on the size and content, this copy gain is expected to be clinically relevant and results in an additional trisomy of the 15q24.1 region.

\subsection{Fluorescence In Situ Hybridization (FISH) Analysis.}

Results of Microarray CGH analysis were confirmed by FISH. FISH analysis of metaphase cells using BAC probes from 7p21.1 (RP11-5G13) and 7p2.1 (RP11-425P5) confirmed the presence of a duplication in the 7p22.1p21.1 region. Metaphase FISH excluded unbalanced translocation. Additional FISH analysis of interphase nuclei using a BAC probe from 15q24.1 (RP11-414J4) confirmed a duplication of the 15q24.1 region.

2.5. Parental Chromosomal Analysis. Subsequent chromosomal analysis of both parents was also completed to determine whether the two interstitial chromosomal gains were de novo in the fetus or from heritable duplication or translocation from a parent. Metaphase FISH analysis revealed normal karyotypes for both parents, with no genetic duplication in either the 7 p22.1p21.1 or the $15 \mathrm{q} 24.1$ region, suggesting that the duplications identified in the fetus were de novo in origin.

2.6. Pregnancy Progression and Postnatal Development. After parental counseling on cytogenetic testing results, the patient elected to continue with the pregnancy. Follow-up imaging showed appropriate fetal growth and additional findings of abnormal calvarial shape suggestive of craniosynostosis and frontal bossing. Fetal echocardiogram at 25-week gestation showed no obvious structural abnormalities.

The pregnancy progressed to 39.2-week gestation, at which time the patient was scheduled for an induction of labor. Patient delivered a male neonate weighing 3,120 g (6 Ibs $14 \mathrm{oz}$ ). Infant had APGAR scores of 7 and 9 at one and five minutes of life, respectively. The neonate was transitioned 
into the Neonatal Intensive Care Unit for evaluation. Initial examination of the neonate revealed low set ears with thick helices, flattened nasal bridge, and ankyloglossia. Although the infant's head shape and size were concerning for craniosynostosis, no formal diagnosis was made. MRI, performed on day two of life, was consistent with partial vermian agenesis and showed hypogenesis of inferior cerebellar vermis with mild fourth ventricular enlargement which communicated with a retrocerebellar cerebrospinal fluid collection (Figure 1(b)). Patient and neonate were discharged on day three of life. At 4 months of age, it became evident that the infant had sensorineural hearing loss of the left ear with unrestricted hearing of contralateral ear, hypotonia, strabismus, and developmental delay. Denver Testing at 9 months of age further characterized the delay. Infant was unable to roll over, had not begun to work for a toy, and did not attempt to feed self. Continued evaluation of the infant throughout development will provide further clarification of the clinical significance and prognosis of these two simultaneous duplications.

\section{Discussion}

Prenatal microarray CGH and FISH analysis of the fetus allowed for the detection of two, previously unreported, simultaneous de novo interstitial duplications resulting in karyotype 46,XY,dup(7)(p22.1-p21.1),dup (15)(q24.1) and partial trisomy of 7p22.1p21.1 and 15q24.1. FISH analysis of the parents demonstrated that both abnormalities were de novo rather than heritable changes.

To our knowledge, the partial trisomy of $7 \mathrm{p} 22.1 \mathrm{p} 21.1$ region seen in the neonate has not been described before. The first report on $7 \mathrm{p}$ interstitial duplication characterized by array CGH was published by Chui and colleagues [1]. Reported findings regarding other $7 \mathrm{p}$ duplications, without involvement of additional chromosomes, describe variable phenotypes, with common features including intellectual disability, hypotonia, craniofacial dysmorphism, skeletal abnormalities, and cardiovascular malformations [1-4]. More specifically, findings suggest that $7 \mathrm{p} 21$ is a critical region for craniofacial dysmorphism and skeletal development [3]. There has been one reported syndrome, Saethre-Chotzen, which is one of the most common autosomal dominant disorders associated with craniosynostosis and is also associated with other craniofacial and limb anomalies. SaethreChotzen syndrome maps to locus 7p21p22 and is caused by a mutation in the TWIST gene, which encodes a basic helixloop-helix transcription factor [5, 6]. TWIST mutations have been described as a result of both duplications and deletions within the gene. These mutations result in impairment of head mesenchyme induction by TWIST and therefore result in craniosynostosis [6]. While there is limited data showing that an increase in the copy number of the TWIST gene may result in craniosynostosis, it is intriguing to speculate that the observed duplications of the 7 p22.1p21.1 region and the corresponding doubling of the copy number of the TWIST gene may lead to similar aberrant gene function and cranial phenotype as reported for the TWIST mutations. When combined, these reports document the potential consequence such a large duplication in $7 \mathrm{p}$ may have on the developing infant.
The simultaneous duplication of $15 \mathrm{q} 24.1$ overlaps a well described microdeletion/microduplication region, $15 q 24.1 \mathrm{q} 24.2$, of chromosome 15. However, what is unique about this case is that the gain identified does not include the entire characterized region commonly described as the smallest region of overlap (SRO). In addition, the duplication breakpoints do not localize to previously described locus control regions (LCRs). For comparison purposes, the proximal duplication breakpoint found in this patient is between $15 \mathrm{q} 24 \mathrm{~A}$ (also referred to as BP4) and 15q24B (BP1), with the distal breakpoint between 15q24B (BP1) and 15q24C as shown by El-Hattab and collaborators $[7,8]$. To date, there have been 15 reported patients with microdeletions and 2 patients with microduplications within this region. The patients with microduplication exhibited a phenotype significant for cognitive impairment, skeletal deformities, and dysmorphic facial features [9]. Together, these reports indicate the potential phenotypical outcomes of such a mutation.

Postnatal evaluation of the neonate has shed some light onto the possible phenotype that may result from these two simultaneous de novo duplications of chromosomal regions 7p22.1p21.1 and 15q24.1. The neonate displayed various craniofacial anomalies including low set ears with thickened helices, flattened nasal bridge, ankyloglossia, and abnormal calvarial shape. Previously published case studies reporting on single duplications in these two chromosomal regions described similar craniofacial anomalies. MRI did confirm the presence of partial vermian agenesis, which can be caused by various genetic anomalies. As the neonate has continued to develop there is additional evidence of developmental delay and unilateral sensorineural hearing loss.

In conclusion, the combination of conventional cytogenetic and molecular cytogenetic analysis and sonographic imaging provided valuable prenatal data on a developing fetus. Microarray CGH and FISH analysis allowed for the identification and characterization of two, previously unreported, simultaneous de novo interstitial duplications of chromosomal regions 7p22.1p21.1 and 15q24.1, resulting in the partial trisomy of 7p22.1p21.1 and 15q24.1. This case well illustrates that microarray CGH and FISH analyses are powerful cytogenetic tools in prenatal diagnosis. It was challenging, however, to provide comprehensive and definitive counseling to the parents on long-term prognosis and perspectives of their infant in the absence of any previous reports documenting genetic aberrations precisely in these two chromosomal regions. Continued observation and monitoring of the infant may provide further insights into the phenotype and the effects caused by these two simultaneous de novo interstitial duplications.

\section{Ethical Approval}

The patient consent to publication in scholarly journal was obtained.

\section{Conflicts of Interest}

The authors declare no conflicts of interest regarding the publication of this paper. 


\section{References}

[1] J. V. Chui, J. D. Weisfeld-Adams, J. Tepperberg, and L. Mehta, "Clinical and molecular characterization of chromosome 7p22.1 microduplication detected by array CGH," American Journal of Medical Genetics Part A, vol. 155, no. 10, pp. 2508-2511, 2011.

[2] E. Papadopoulou, S. Sifakis, C. Sarri et al., "A report of pure 7p duplication syndrome and review of the literature," American Journal of Medical Genetics Part A, vol. 140, no. 24, pp. 28022806, 2006.

[3] E. Preiksaitiene, J. Kasnauskiene, Z. Ciuladaite, B. Tumiene, P. C. Patsalis, and V. Kučinskas, "Clinical and molecular characterization of a second case of 7p22.1 microduplication," American Journal of Medical Genetics Part A, vol. 158, no. 5, pp. 1200-1203, 2012.

[4] L. Zahed, T. Pramparo, C. Farra, M. Mikati, and O. Zuffardi, "A patient with duplication (7)(p22.1pter) characterized by arrayCGH," American Journal of Medical Genetics Part A, vol. 143, no. 2, pp. 168-171, 2007.

[5] T. D. Howard, W. A. Paznekas, E. D. Green et al., "Mutations in TWIST, a basic helix-loop-helix transcription factor, in SaethreChotzen syndrome," Nature Genetics, vol. 15, no. 1, pp. 36-41, 1997.

[6] V. E. Ghouzzi, M. L. Merrer, F. Perrin-Schmitt et al., "Mutations of the TWIST gene in the Saethre-Chotzen syndrome," Nature Genetics, vol. 15, no. 1, pp. 42-46, 1997.

[7] A. W. El-Hattab, F. Zhang, R. Maxim et al., "Deletion and duplication of 15q24: Molecular mechanisms and potential modification by additional copy number variants," Genetics in Medicine, vol. 12, no. 9, pp. 573-586, 2010.

[8] A. W. El-Hattab, Z. Ou, S.-H. L. Kang et al., "Redefined genomic architecture in 15q24 directed by patient deletion/ duplication breakpoint mapping," Human Genetics, vol. 126, no. 4, pp. 589602, 2009.

[9] A. B. Kiholm Lund, H. D. Hove, and M. Kirchhoff, "A 15q24 microduplication, reciprocal to the recently described $15 \mathrm{q} 24$ microdeletion, in a boy sharing clinical features with 15q24 microdeletion syndrome patients," European Journal of Medical Genetics, vol. 51, no. 6, pp. 520-526, 2008. 


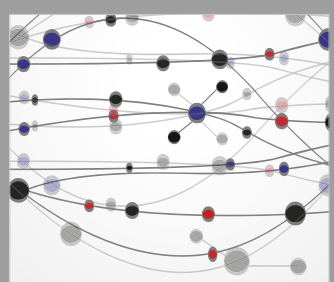

The Scientific World Journal
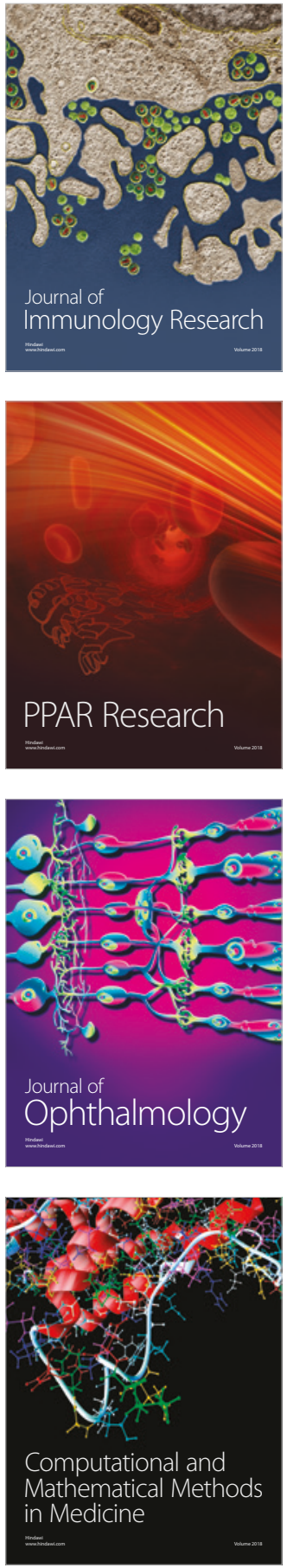

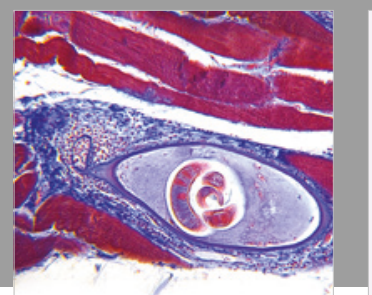

Gastroenterology Research and Practice

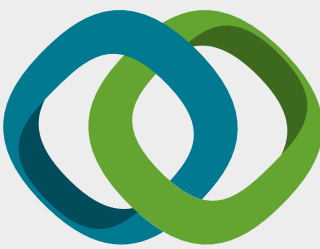

\section{Hindawi}

Submit your manuscripts at

www.hindawi.com
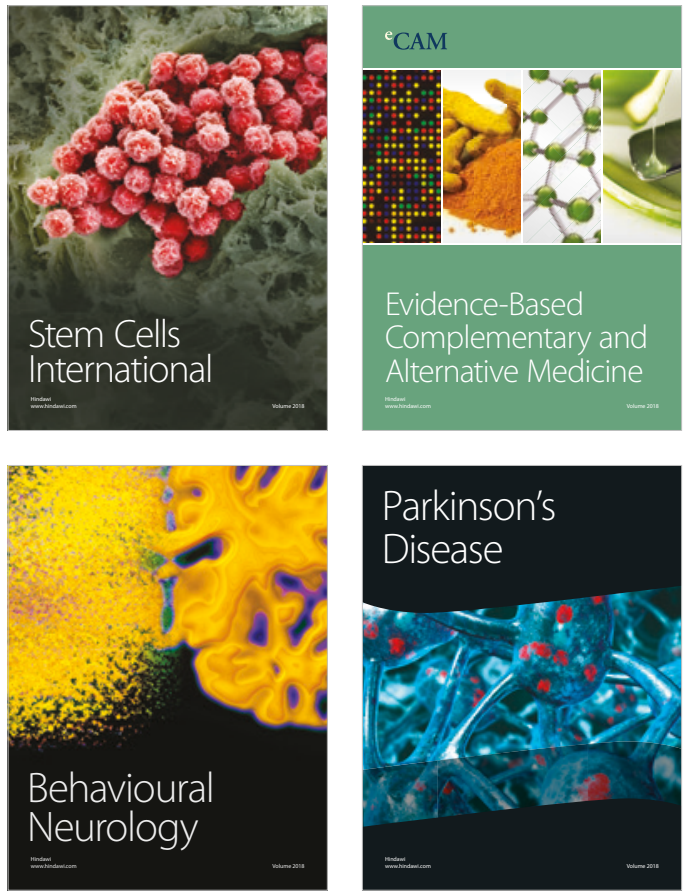

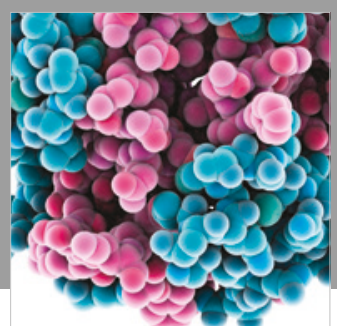

ournal of

Diabetes Research

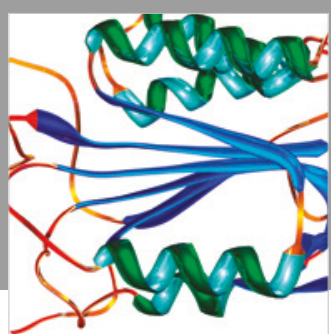

Disease Markers
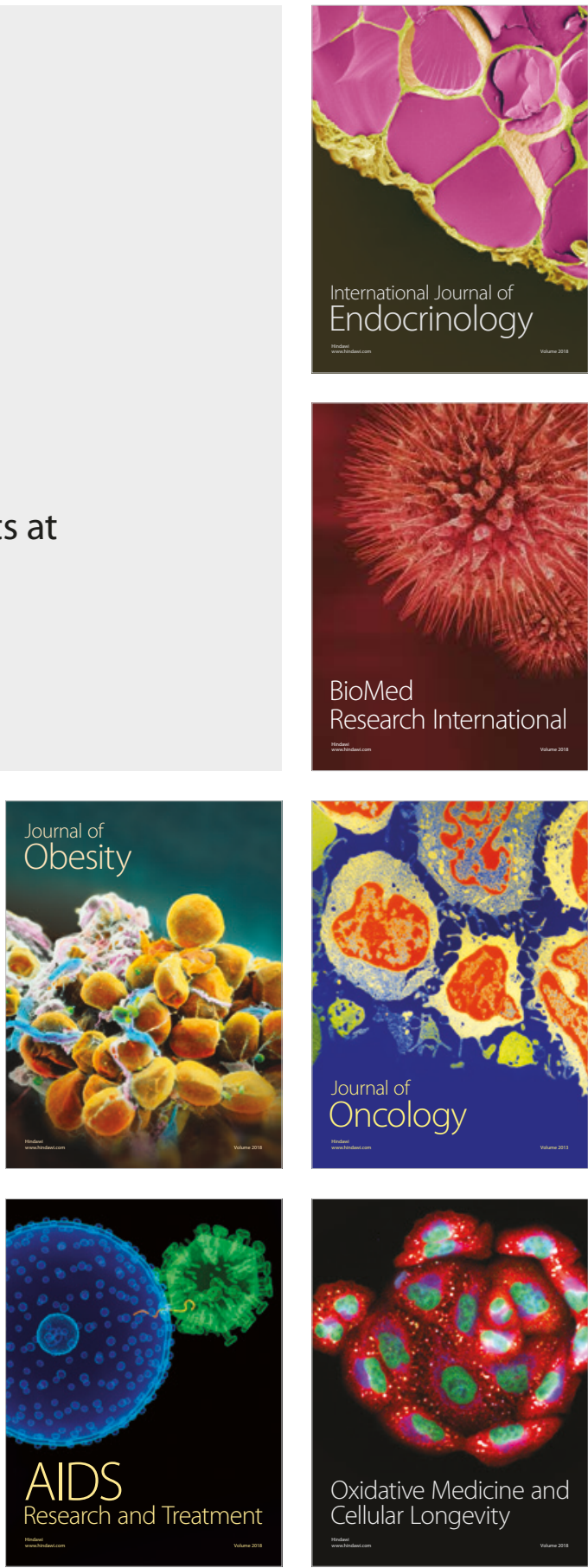